\title{
A Comparative Study of Prostate Cancer Detection Rate Between Transperineal COG-TB and Transperineal FUS-TB in Patients with PSA $\leq 20 \mathrm{ng} / \mathrm{mL}$
}

\author{
Linghui Liang, MD, ${ }^{*}$ Yifei Cheng, MD, ${ }^{*}$ Feng Qi, MD, ${ }^{*}$ Lei Zhang, MD, Dongliang Cao, MD, \\ Gong Cheng, MD, PhD, and Lixin Hua, MD, PhD
}

\begin{abstract}
Background: The combination of prebiopsy MRI and transperineal targeted biopsies is being increasingly used to obtain tissues from patients with suspected prostate cancer (PCa).

Objective: To investigate the difference in PCa detection rate between transperineal cognitive fusion TB (COGTB) and transperineal software fusion TB (FUS-TB).

Participants: The present study included 163 male patients with suspected PCa who had not undergone prostate biopsy, had a prostate-specific antigen (PSA) level of $\leq 20 \mathrm{ng} / \mathrm{mL}$, and had been examined by bi-parameter MRI and confirmed to have prostate nodules by prostate imaging reporting and data system version 2 (PI-RADS V2) scores $\geq 3$ (from December 3, 2018 to October 7, 2019).

Intervention: Seventy-one patients underwent transperineal COG-TB, and 92 patients underwent transperineal FUS-TB. The detection rate of the first four needles was compared.

Results: No significant difference was found in the overall detection rate of PCa between COG-TB and FUS-TB $(60.56 \%$ vs $51.08 \%, p=0.228)$. This result was consistent even after stratifying by PI-RADS score. There was also no significant difference between COG-TB and FUS-TB in the detection rate of clinically significant PCa $(p=0.641)$. Moreover, COG-TB and FUS-TB showed no difference in the detection rate of PCa with different Gleason scores.

Conclusions: In patients with suspected PCa with PSA $\leq 20 \mathrm{ng} / \mathrm{mL}$ and PI-RADS $\geq 3$, FUS-TB was comparable to $\mathrm{COG}-\mathrm{TB}$ in the detection rate of $\mathrm{PCa}$.
\end{abstract}

Keywords: prostate cancer, cognitive fusion, software fusion, transperineal targeted biopsy, magnetic resonance imaging

\section{Introduction}

$\mathbf{P}$ Rostate CANCER (PCA) Is the second most common cancer and the fifth leading cause of cancer-related death in men worldwide. PCa is the most frequently diagnosed cancer in men in more than $50 \%$ of the countries in the world, especially in the Americas, Northern and Western Europe, Australia, and much of sub-Saharan Africa. With regard to mortality rate, $\mathrm{PCa}$ is the leading cause of cancer-related death in men in 46 countries. ${ }^{1}$

With regard to tumor characteristics, the progression of $\mathrm{PCa}$ in the early stage is slow, and patients with PCa at this stage are often asymptomatic. This is because lesions are limited to the prostate, which makes it difficult to be detected by digital rectal examination (DRE). Once $\mathrm{PCa}$ progresses to the advanced stage, it develops rapidly and tends to metastasize. Hence, promoting early diagnosis of $\mathrm{PCa}$ is the first step to improve the survival rate of patients with PCa.

In recent years, prebiopsy MRI played an increasingly significant role in the early diagnosis of $\mathrm{PCa}^{2}$ MRI guided biopsy (targeted biopsy [TB]) had been paid more and more attention in clinical practice. ${ }^{3}$ TB could be carried out by different approaches such as cognitive fusion TB (COG-TB) and software fusion TB (FUS-TB). COG-TB and FUS-TB have their own strengths and weaknesses. COG-TB has the advantages of simplicity, rapidity, and no requirement of additional equipment apart from MRI and conventional transrectal ultrasound (TRUS) facilities. However, regarding the clinical work for COG-TB, clinicians should not only master prostate ultrasound examination technology but also they should be proficient in reading multiparametric MRI

Department of Urology, First Affiliated Hospital of Nanjing Medical University, Nanjing, China.

*These authors contributed equally to this article and should be considered as co-first authors. 
(mpMRI) sequences. The area below the learning curve is wide, the learning cycle is long, and different operators have individual biases and limitations in accurately performing COG-TBs. In contrast, FUS-TB relies less on professional knowledge than COG-TB. The lesions are located using the software system and determined by the combination of mpMRI and ultrasound imaging; this compensates for the disadvantage that COG-TB cannot precisely identify the lesions of painless $\mathrm{PCa}{ }^{4}$ On a global scale, however, because of the differences in regions, races, and individuals, it is difficult to use a common model for the prostate. Moreover, the software uses many variables, which make it difficult to determine a unified coefficient.

Generally, prostate biopsies are divided into transrectal prostate biopsy and transperineal prostate biopsy depending on the puncture site. ${ }^{5}$ Transperineal prostate biopsy has a lower risk of infection and rectal bleeding than transrectal prostate biopsy. ${ }^{6}$

To date, few studies have compared and estimated the detection rate of COG-TB and FUS-TB. Hence, in the present study, we aimed to report the clinical findings and outcomes for 163 patients who underwent prebiopsy MRI along with transperineal COG-TB or FUS-TB to determine the advantages and disadvantages of these techniques and the optimal approach to perform prostate biopsies.

\section{Methods}

\section{Study population}

The present study was a retrospective analysis of 200 patients with suspected PCa who had not underwent prostate biopsy, had prostate-specific antigen (PSA) level of $\leq 20 \mathrm{ng} / \mathrm{mL}$, and had been examined by bi-parameter MRI (bpMRI) and were confirmed to have prostate nodules based on prostate imaging reporting and data system version 2 (PIRADS V2) scores of $\geq 3$ from December 3, 2018 to October 7, 2019. All the patients included in this research were biopsy naive, with PSA concentrations $\leq 20 \mathrm{ng} / \mathrm{mL}$ and accepted bpMRI examination in our center. So, 37 patients were excluded before analysis, including 29 patients with MRIs performed in other institutions and 8 were not biopsy naive. Finally, 71 patients who underwent transperineal COG-TB and 92 who underwent transperineal FUS-TB were included (Fig. 1). All the patients had been examined by prebiopsy MRI scans in the same hospital because of elevated PSA level or abnormal DRE result. Twenty-two patients with positive COG-TB biopsies and 47 patients with positive FUS-TB underwent surgery. Finally, the pathologic results of these patients were recorded. This study was approved by the Institutional Review Board and the First Affiliated Hospital of Nanjing Medical University Ethics Committee, and informed consent was obtained from all patients and volunteers.

\section{MRI protocol}

The bpMRI was used for prebiopsy MRI scan. ${ }^{7}$ All MRI scans were performed at $3.0 \mathrm{~T}$ with axial $\mathrm{T} 1$-weighted images (T1WI), multiplane (Axial, coronal, and sagittal) T2-weighted images (T2WI), and axial diffusion weighted imaging (DWI) (measured b-values of 0.400 and $800 \mathrm{sec}-$ onds $/ \mathrm{mm}^{2}$, calculated b-value of $2000 \mathrm{~seconds} / \mathrm{mm}^{2}$ ), but

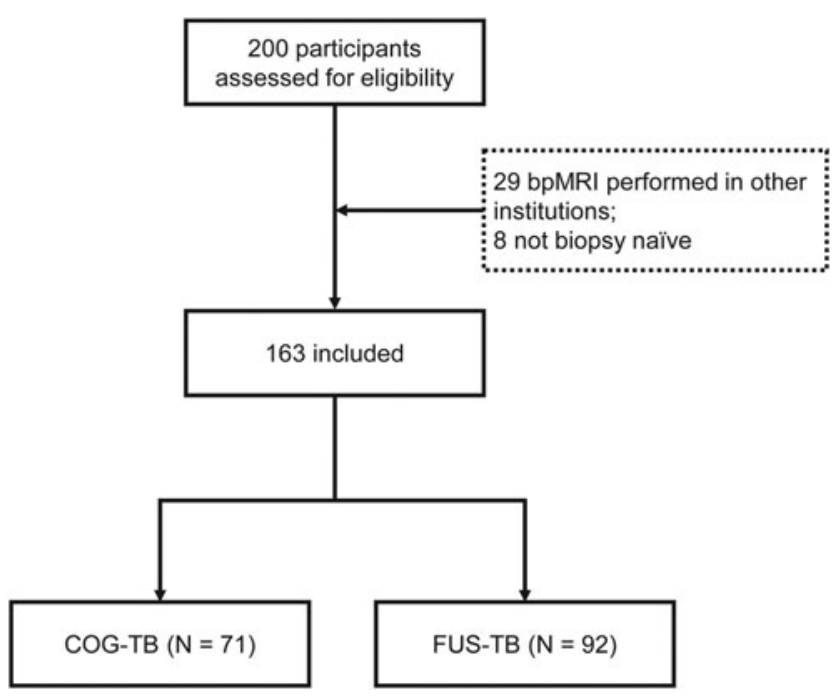

FIG. 1. Flow chart of criteria for inclusion in the present study.

without dynamic contrast enhancement (DCE). ${ }^{8}$ The prostate nodules found by bpMRI were evaluated by PI-RADS V2 scores by the deputy chief or senior physicians. ${ }^{9}$ The lesions were located in detail before the puncture (lesions' position, diameter, distance from the bladder neck, and distance from the dorsal margin of the prostate). If the lesion was located in the peripheral zone, DWI of the pelvis and its apparent diffusion coefficient were used to determine the target lesion. ${ }^{10}$ If the lesion appeared in the transitional zone, the target lesion was determined by coronal or sagittal T2W imaging of the pelvis. A single fellowshiptrained radiologist (familiar with PI-RADS version 2 guideline and having the experience in reading prostate MRI images for more than 10 years) with prostate imaging evaluated all the examinations based on a previously published methodology for the interpretation and reporting of prostate MRI scans.

\section{COG-TB and FUS-TB}

In COG-TB, we located the lesions in three dimensions, namely coronal, sagittal, and horizontal planes, on MRI. The location of the lesion determined by MRI was then compared with the hypoechoic image of TRUS to determine the puncture site. ${ }^{11}$

In terms of FUS-TB, first, the MRI image of prostate was imported into the software platform (Predictive Fusion Software; BK medical, Capital Region, Denmark). Then, the prostate shape and lesions were automatically drawn and registered with TRUS image by the software. Under the guidance of TRUS, real-time tumor location was performed by computer software.

\section{Biopsy procedure}

Two experienced urologists (with experience in transperineal standard biopsy for more than 1 year and familiar in prostatic MRI) performed TRUS-guided transperineal prostate biopsy in the operation room. The patient adopted the lithotomy position and underwent routine disinfection and 
perineal local anesthesia. The patient was placed in the lithotomy position. Perineal skin was disinfected using an iodophor solution. First, local anesthetic is injected in the areas (perineal skin) that will be subsequently traversed for the biopsy. Then, under TRUS guidance $20 \mathrm{cc}$ (10 cc per lobe) of lidocaine $2 \%$ were injected at the posterior prostate apex on the capsule surface. After peri-prostatic injection, while exiting the needle (without further puncture) also injected in perineal muscular planes and subcutaneous perineal planes was induced, and the rectal B-ultrasound probe was then fixed on the puncture frame. After the probe was placed on the rectum, the puncture template was fixed on the puncture frame. Combined with the cognitive results or software digital fusion, the puncture frame walking device was used to press the transverse section of the urethral orifice at $0.5 \mathrm{~cm}$. The rectal probe was retreated at the same distance from the neck of the bladder to the cross-section where the lesion was located, and the center of the tumor on the X-and Y-axes was determined by the ultrasound localization system. The needle was then entered from the corresponding puncture hole of the template to determine the center of the tumor by focusing on the $\mathrm{X}$ - and $\mathrm{Y}$-axes using the ultrasound localization system. TB was then performed. Targeted biopsies were performed with four needles for each target (per index lesion). If there are more than two targets, the one with a higher PI-RADS score was analyzed. Details of the type and dose of the anesthetic used were not obtained at the time of data collection.

\section{Statistical analyses}

SPSS version 22.0 (IBM Corp., Armonk, NY) statistical software was used to analyze all the data. Differences in demographic distributions between patients who underwent COG-TB or FUS-TB were compared by Student's $t$-test and the chi-square test for continuous variables and categorical variables, respectively. The chi-square test was used to compare differences between the detection rates of $\mathrm{PCa}$, clinically significant PCa (csPCa, defined as Gleason score $\geq 3+4$ ), and the upgrade rates of Gleason scores in the two groups. Patients with different Gleason scores were compared using the rank sum test. All analyses were two sided, and $p<0.05$ was considered to be statistically significant.

\section{Results}

\section{Baseline characteristics}

The mean (standard deviation) age, median (interquartile range $[\mathrm{IQR}]$ ) body mass index (BMI), median (IQR) PSA, and median (IQR) MRI prostate volume of the COG-TB group were 69.17 (9.18) years, $23.87(3.30) \mathrm{kg} / \mathrm{m}^{2}, 7.66$ $(5.38) \mathrm{ng} / \mathrm{mL}$, and $34.74(29.41) \mathrm{cm}^{3}$, respectively, and those of the FUS-TB group were 67.59 (8.45) years, 23.68 (3.30) $\mathrm{kg} / \mathrm{m}^{2}, 8.03(6.18) \mathrm{ng} / \mathrm{mL}$, and $34.94(26.31) \mathrm{cm}^{3}$, respectively (Table 1). In addition, 34 (47.9\%) patients in the COGTB group and $42(45.7 \%)$ patients in the FUS-TB group were reported to have suspicious DRE findings. There was no significant difference in all these characteristics between the two groups.

\section{Comparison of the detection rate between COG-TB and FUS-TB}

Overall, $60.56 \%$ of the patients who underwent COG-TB and $51.08 \%$ of those who underwent FUS-TB were found to have $\mathrm{PCa}$. There was no significant difference in the detection rate of PCa between COG-TB and FUS-TB $(p=0.228$, Fig. 2). In addition, $39.43 \%$ and $35.87 \%$ of the patients were found to have csPCa by COG-TB and FUS-TB, respectively. No significant difference in the detection of csPCa ( $p=0.641$, Fig. 3) was found between the two groups.

According to the PSA level, the patients in the two groups were divided into PSA $\leq 10 \mathrm{ng} / \mathrm{mL}$ and $10<\mathrm{PSA} \leq 20 \mathrm{ng} / \mathrm{mL}$ subgroups. Subgroup analysis showed no significant difference in the detection rate of PCa between COG-TB and FUSTB in patients with PSA $\leq 10 \mathrm{ng} / \mathrm{mL}(p=0.347)$ and patients with $10 \mathrm{ng} / \mathrm{mL}<$ PSA $\leq 20 \mathrm{ng} / \mathrm{mL}$ ( $p=0.174$, Table 2$)$. Furthermore, we compared the difference in the detection rate between COG-TB and FUS-TB stratified by the PI-RADS scores. As shown in Table 2, the detection rates of PCa in patients with PI-RADS score of 3, 4, and 5 were $40.0 \%$, $74.07 \%$, and $77.78 \%$ for COG-TB and $22.45 \%, 77.42 \%$, and $100 \%$ for FUS-TB, respectively. No notable difference was found ( $p=0.096,0.766$, and 0.130 for PI-RADS score of 3, 4, and 5, respectively, Fig. 4). Similar results were noted for the detection rate of csPCa $(p=0.776,0.873$, and 0.689 for PIRADS score of 3, 4, and 5, respectively).

\section{Patients with PCa with different Gleason scores and upgrading}

We also analyzed the proportion of Gleason scores in the two groups of patients with PCa. The Gleason scores for $\mathrm{PCa}$ in the COG-TB group were 15 Gleason $=3+3$, 9 Gleason $=3+4,8$ Gleason $=4+3,9$ Gleason $=4+4$, and 2 Gleason $=4+5$, and in the FUS-TB group, the scores were 16 Gleason $=3+3,13$ Gleason $=3+4,8$ Gleason $=4$ $+3,7$ Gleason $=4+4$, and 3 Gleason $=4+5$. No significant difference was noted between these groups $(p=0.792$,

Table 1. Baseline Characteristics

\begin{tabular}{lccr}
\hline Value & COG-TB & FUS-TB & $\mathrm{p}$ \\
\hline Age $(\mathrm{year})$ & $69.17 \pm 9.18$ & $67.59 \pm 8.45$ & 0.310 \\
BMI $\left(\mathrm{kg} / \mathrm{m}^{2}\right)$ & $23.87(16.65-30.07)$ & $23.68(16.20-35.38)$ & 0.690 \\
PV $\left(\mathrm{cm}^{3}\right)$ & $34.74(12.48-167.40)$ & $34.94(17.58-111.45)$ & 0.768 \\
PSA $(\mathrm{ng} / \mathrm{mL})$ & $7.66(0.67-18.81)$ & $8.03(0.66-19.78)$ & 0.172 \\
PSAD & $0.22(0.05-0.11)$ & $0.23(0.04-0.17)$ & 0.780 \\
DRE $+(\%)$ & $34(47.89)$ & $42(45.65)$ & 0.777 \\
\hline
\end{tabular}

$\mathrm{BMI}=$ body mass index COG-TB $=$ cognitive fusion $\mathrm{TB} ; \mathrm{FUS}-\mathrm{TB}=$ software fusion $\mathrm{TB} ; \mathrm{PSA}=$ prostate-specific antigen; $\mathrm{PSAD}=\mathrm{PSA}$ density; $\mathrm{PV}=$ prostate volume. 
Stratified PI-RADS of patients

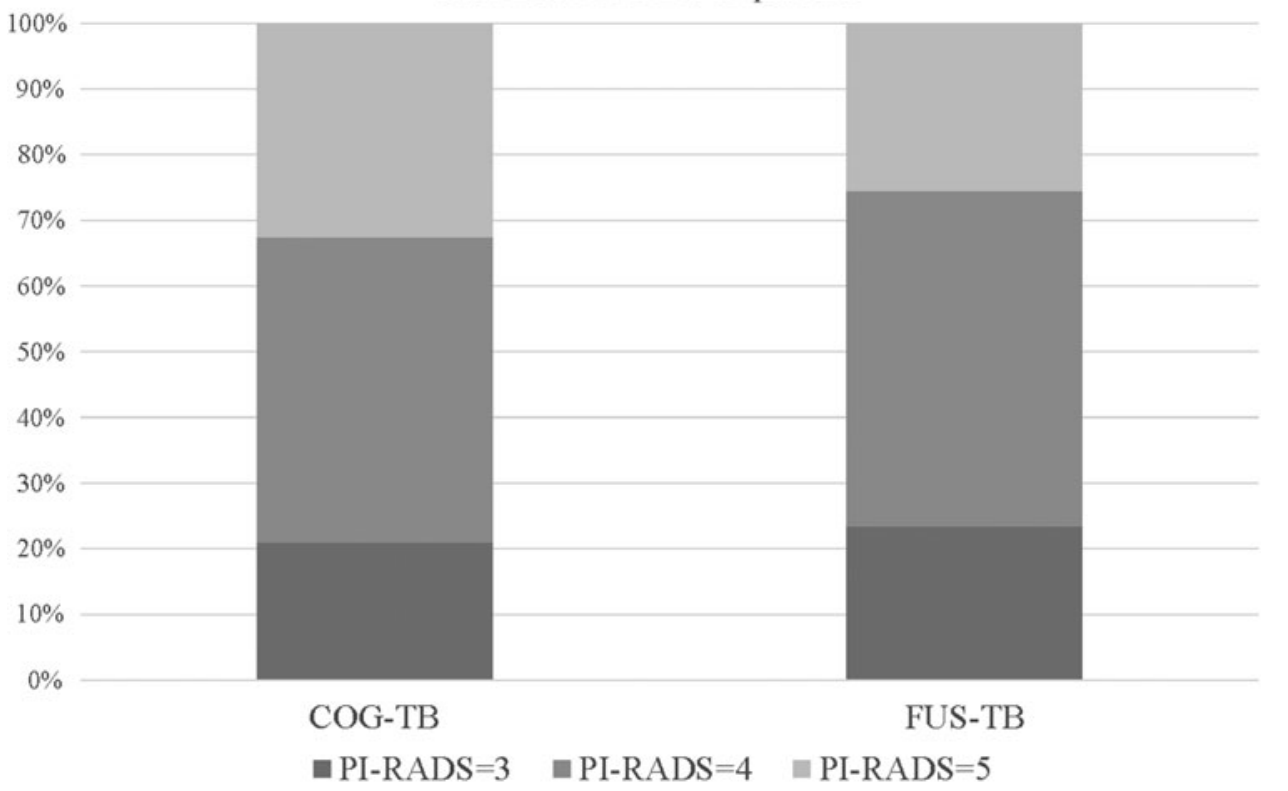

FIG. 2. Comparison of the detection rate of PCa between stratified PI-RADS of COG-TB and FUS-TB. COG-TB $=$ cognitive fusion TB; FUS-TB $=$ software fusion $\mathrm{TB} ; \mathrm{PCa}=$ prostate cancer; PI-RADS = prostate imaging reporting and data system.
Table 2), thus indicating no difference in the composition of the population of the two groups.

Finally, we analyzed whether the preoperative Gleason scores were upgraded to higher ones in the pathologic results after radical prostatectomy. We found that preoperative Gleason score of 8 (36.4\%) patients with PCa diagnosed by COG-TB and those of $11(23.4 \%)$ patients diagnosed by FUS-TB upgraded to higher ones, but showed no significant difference $(p=0.552)$.

\section{Complications}

In our cohort, postbiopsy fevers occurred in two patients who accepted COG-TB and one who underwent FUS-TB. In addition, bacteremia occurred in one patient after FUS-TB.
None of the patients developed severe bleeding, dysuria, vasovagal reactions, or other complications that required to be addressed.

\section{Discussion}

Thus far, patients with abnormally elevated PSA level, prostate nodules, and abnormal lesions found by imaging systems are often subjected to transrectal or perineal system plus targeted prostate biopsies to determine the presence of PCa. ${ }^{12}$ The combination of these two techniques could improve the detection rate of $\mathrm{PCa}$, thus functioning as the best prostate biopsy approach at present. ${ }^{13}$ Because not all PCas show hypoechoic nodules under TRUS, relying on ultrasound alone would lead to missed diagnosis in some cases. MRI is

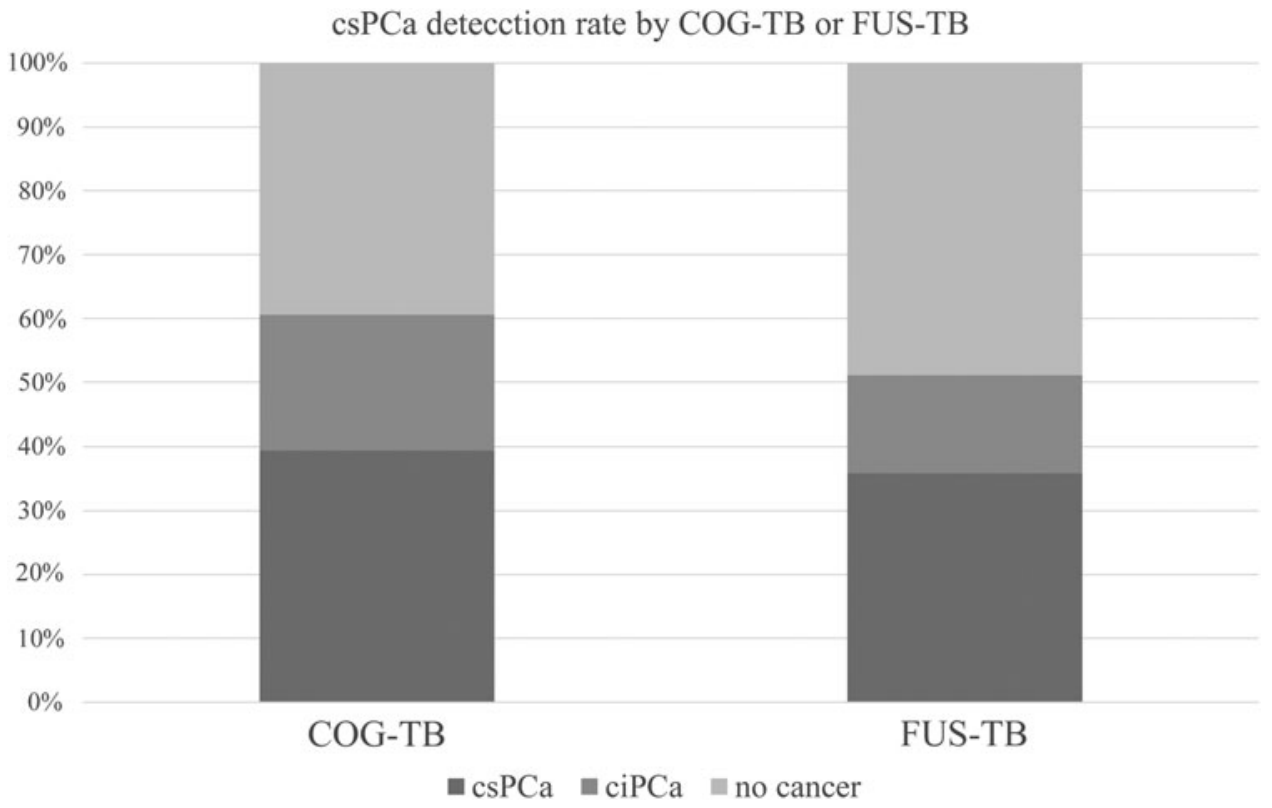

FIG. 3. Comparison of the csPCa detection rate between COG-TB and FUS-TB. csPCa $=$ clinically significant prostate cancer. 
Table 2. Comparison Between Cognitive Fusion Biopsy and SOFTwARE Fusion Biopsy

\begin{tabular}{lccc}
\hline Outcome & $\begin{array}{c}\text { COG-TB } \\
(\mathrm{n}=71)\end{array}$ & $\begin{array}{c}F U S-T B \\
(\mathrm{n}=92)\end{array}$ & $\mathrm{p}^{\mathrm{a} / \mathrm{b}}$ \\
\hline $\begin{array}{l}\text { PCa detection rate } n(\%) \\
\text { Overall }\end{array}$ & $43(60.56)$ & $47(51.08)$ & 0.228 \\
PSA, ng/mL & $26(52.00)$ & $24(42.86)$ & 0.347 \\
0-10 & $17(80.95)$ & $23(63.89)$ & 0.174 \\
10-20 & & & \\
PI-RADS & $9(30.00)$ & $11(22.45)$ & 0.454 \\
3 & $20(74.07)$ & $24(77.42)$ & 0.766 \\
4 & $14(77.78)$ & $12(100.00)$ & 0.130 \\
5 & & & \\
csPCa detection rate $n(\%)$ & $28(39.43)$ & $33(35.87)$ & 0.641 \\
Overall & & & \\
PI-RADS & $5(17.86)$ & $5(15.15)$ & 0.776 \\
3 & $15(53.57)$ & $17(51.52)$ & 0.873 \\
4 & $8(28.57)$ & $11(33.33)$ & 0.689 \\
5 & & & \\
Gleason score & 15 & 16 & 0.792 \\
$3+3$ & 9 & 13 & \\
$3+4$ & 8 & 8 & \\
$4+3$ & 9 & 7 & \\
$4+4$ & 2 & 3 & \\
$4+5$ & $8(36.36)$ & $11(23.40)$ & 0.552 \\
Upgrade rate $n(\%)$ & & & \\
\hline
\end{tabular}

${ }^{\mathrm{a}} \mathrm{PCa} / \mathrm{cs} \mathrm{PCa}$ detection rate uses chi-square test.

${ }^{\mathrm{b}}$ Gleason score uses Kruskal-Wallis test.

Criteria for csPCa: a Gleason score of $\geq 3+4$

csPCa $=$ clinically significant prostate cancer; $\mathrm{PI}-\mathrm{RADS}=$ prostate imaging reporting and data system.

more effective in the diagnosis of PCa lesions, and its sensitivity and specificity for the diagnosis of PCa are relatively high. ${ }^{14}$ MRI has gained popularity as the best imaging method for the detection of suspicious PCa. Schoots and coworkers ${ }^{15}$ conducted Systematic review and meta-analysis of evidence regarding the diagnostic benefits of MRI-TBX $v s$ TRUS-PBX in detection of overall $\mathrm{PCa}$ (primary objective) and $\mathrm{csPCa} /$
ciPCa (secondary objective). They found that MRI-TBx had a higher rate of detection of significant $\mathrm{PCa}$ and a lower rate of detection of insignificant PCa compared with TRUS-Bx. Review by Wegelin and associates ${ }^{16}$ showed that MRI-guided biopsy detects more csPCa and less ciPCa compared with systematic biopsy in men at risk for PCa. Delongchamps and colleagues $^{17}$ found that TB alone could improve cancer detection over random biopsies, decreased the detection rate of micro focal cancer, and increased the detection rate of cancer with a Gleason score of $>6$. Therefore, MRI can provide more meaningful data for COG-TB and FUS-TB in detecting suspicious lesions, thus providing puncture targets and improving the detection rate of PCa. Meanwhile, given the limited role of DCE, there is growing interest in performing prostate MRI without DCE, a procedure termed "biparametric MRI." A number of recent studies have reported data that support the value of bpMRI for the detection of csPCa in biopsy-naive men and those with a prior negative biopsy. ${ }^{18}$

In the present study, we performed biopsies in male patients with suspicious PCa with PSA $\leq 20 \mathrm{ng} / \mathrm{mL}$ and positive MRI findings. We found no significant difference between COGTB and FUS-TB. And our detection of PCa $(60.56 \%$ and $51.08 \%)$ is not much different from MRI-FIRST ${ }^{19}(59 \%)$ and PRECISION $^{20}(47 \%)$. Oderda and coworkers ${ }^{21}$ found that elastic fusion biopsies performed with Koelis achieve an increased per patient and per lesion cancer detection rate compared to COG-TB, especially in the case of lesions $\leq 10 \mathrm{~mm}$. However, it should be noted that the sample size was relatively small and this was a single-center study, which might lead to certain limitations. Hence, a larger sample size and multicenter studies are needed for the validation of our results.

Yamada and associates ${ }^{22}$ grouped 298 patients with suspected PCa into the COG-TB group and the FUS-TB group. The following results were obtained: the any-cancer detection rate was $79.6 \%$ and $84.8 \%$ in the COG-TB and FUS-TB groups, respectively ( $p=0.516$ ), and the csPCa detection rate was $72.5 \%$ and $75.7 \%$ in the COG-TB and FUS-TB groups, respectively $(p=0.498)$. Monda and associates ${ }^{23}$ reported that the rates of upstaging or missing csPCa with FUS-TB were
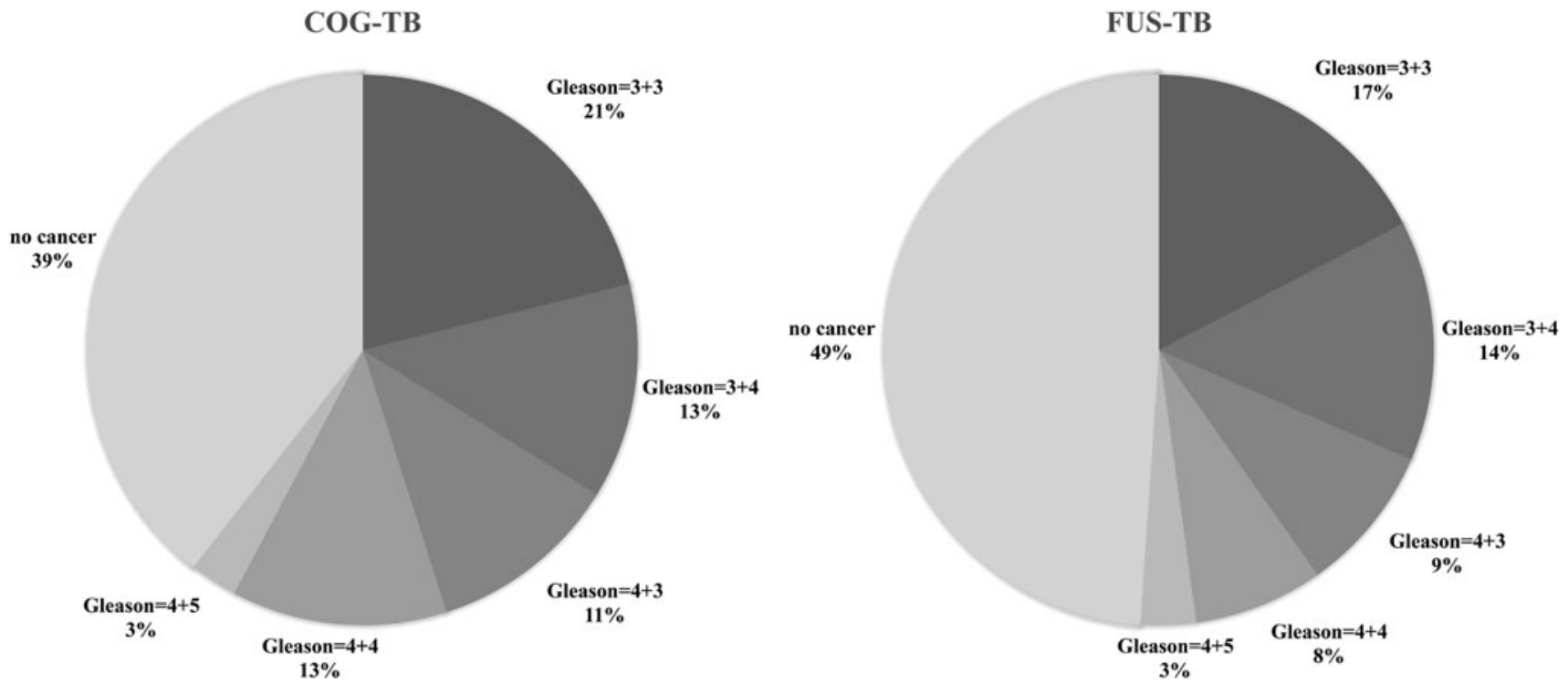

FIG. 4. Percentage of PCa with different Gleason scores from COG-TB and FUS-TB. 
not significantly different from those of COG-TB $(p=0.172)$; moreover, the risk of missing csPCa was not different between FUS-TB and COG-TB in logistic regression (odds ratio $=0.55, p=0.106$ ). No significant difference in biopsy outcomes was observed between FUS-TB and COG-TB $(p=0.171)$. In the institutional experience of these authors, no significant difference was observed between the diagnostic ability of COG-TB and FUS-TB for detecting csPCa. Baco and coworkers ${ }^{24}$ randomly grouped 175 patients with suspected PCa who had not undergone prostate puncture into the MRI group and the control group. The results showed that the diagnostic rates were $59 \%$ and $54 \%(p=0.4)$, respectively. The diagnostic rates for clinically significant tumors were $44 \%$ and $49 \%$ ( $p=0.5)$, respectively. No significant difference was noted between the two groups. Puech $\mathrm{P}$ and associates ${ }^{25}$ found that in $79 \mathrm{MR}$ imaging targets, the positivity for cancer was $47 \%(n=37)$ with COG-TB and 53\% $(n=42)$ with FUS-TB $(p=0.16)$. No significant difference in TB guidance was observed between FUS-TB and COG-TB. The findings of all these studies are consistent with the results of our present study.

Compared to COG-TB, the procedure of FUS-TB is simple, and the learning cost is low. Because the prostate model is already established in the software, FUS-TB depends less on professional knowledge than COG-TB. The software system enables one to localize the lesions. By combining MRI and ultrasound imaging, the location and size of lesions can be determined to compensate for the disadvantage that COG-TB cannot identify clinically insignificant painless PCa lesions. However, FUS-TB requires the organization to purchase the copyright and rights to use the software, which are relatively expensive. Consequently, the utility of FUS-TB is limited. ${ }^{26}$ However, the process of using software fusion biopsies also enhances the MRI reading capability of the clinicians.

The COG-TB technique is free from software fusion of images, and although its equipment cost is low, the operation is simple and rapid, and it can be easily popularized in clinics; the conversion of MRI images to TRUS images may lead to subjective errors. ${ }^{27}$ Therefore, physicians should not only master the prostate ultrasound examination technology but also they should become proficient in reading MRI sequences; thus, COG-TB has a wide area under the learning curve and a long learning cycle. With the continuous simplification of the rules and regulations of the center and the continuous improvement of the biopsy process, these technologies are transferring to the outpatient setting. It can make the biopsy process more economical and efficient. According to our recent implementation results, this behavior is feasible and advocated.

The detection rate of $\mathrm{PCa}$ in the present study was higher compared with $35 \%$ and $40 \%$ reported by global studies with large samples of $\mathrm{PCa}{ }^{28}$ This difference may be related to the basic clinical conditions of the patients included in the experimental group and the application of COG-TB or FUSTB. The present study is a randomized controlled trial and a retrospective analysis as well. Moreover, the sample size of the present study was relatively small, and it was a singlecenter study. Considering these aspects and the strict inclusion criteria of the present study, the generalization of our results might be limited.

In summary, FUS-TB has no advantage over COG-TB in patients with PSA $\leq 20 \mathrm{ng} / \mathrm{mL}$ and PI-RADS $\geq 3$; however, it is necessary to improve the clinical experience of biopsy operators, the cognition of the prostate stereoscopic anatomy, and the MRI recognition capability.

\section{Conclusion}

Our present study revealed no significant difference in the detection rate of $\mathrm{PCa}$ between transperineal COG-TB and FUS-TB.

\section{Informed Consent}

All patients have signed informed consent.

\section{Authors' Contributions}

L.L., Y.C., F.Q.: Project development, Data Collection, Article writing; D.C., L.Z.: Data collection; L.H., G.C.: Project development.

\section{Author Disclosure Statement}

No competing financial interests exist.

\section{Funding Information}

No funding was received for this article.

\section{References}

1. Bray F, Ferlay J, Soerjomataram I, Siegel RL, Torre LA, Jemal A. Global cancer statistics 2018: GLOBOCAN estimates of incidence and mortality worldwide for 36 cancers in 185 countries. CA Cancer J Clin 2018;68:394-424.

2. Gayet M, van der Aa A, Beerlage HP, Schrier BP, Mulders $\mathrm{PF}$, Wijkstra $\mathrm{H}$. The value of magnetic resonance imaging and ultrasonography (MRI/US)-fusion biopsy platforms in prostate cancer detection: A systematic review. BJU Int 2016;117:392-400.

3. Norris JM, Kinnaird A, Margolis DJ, Padhani AR, Walz J, Kasivisvanathan V. Developments in MRI-targeted prostate biopsy. Curr Opin Urol 2020;30:1-8.

4. Mukendi AM, Van Den Berg E, Pather S, Padayachee RS. Metachronous or synchronous male breast and prostate cancers a duality to lookout for. F1000Res 2018;7:1825.

5. He BM, Chen R, Shi ZK, et al. Trans-perineal templateguided mapping biopsy vs. freehand trans-perineal biopsy in Chinese patients with PSA $<20 \mathrm{ng} / \mathrm{ml}$ : similar cancer detection rate but different lesion detection rate. Front Oncol 2019;9:758.

6. Borghesi M, Ahmed H, Nam R, et al. Complications after systematic, random, and image-guided prostate biopsy. Eur Urol 2017;71:353-365.

7. Morote J, Celma A, Planas J. Re: Lars Boesen, Nis Norgaard, Vibeke Logager, et al. Prebiopsy biparametric magnetic resonance imaging combined with prostatespecific antigen density in detecting and ruling out Gleason 7-10 prostate cancer in biopsy-naive men. Eur Urol Oncol 2019;2:311-319.

8. Mussi TC, Yamauchi FI, Tridente CF, et al. Interobserver agreement of PI-RADS v. 2 lexicon among radiologists with different levels of experience. J Magn Reson Imaging 2020;51:593-602.

9. Khoo CC, Eldred-Evans D, Peters M, et al. Likert vs. PIRADS v2: A comparison of two radiological scoring systems for detection of clinically significant prostate cancer. BJU Int 2020;125:49-55.

10. Luczynska E, Heinze-Paluchowska S, Domalik A, et al. The utility of diffusion weighted imaging (DWI) using 
apparent diffusion coefficient (ADC) values in discriminating between prostate cancer and normal tissue. Pol J Radiol 2014;79:450-455.

11. Wang HF, Chen R, He BM, et al. Initial experience with a novel method for cognitive transperineal magnetic resonance imaging-targeted prostate biopsy. Asian J Androl 2020;22:432-436.

12. Ezquer A, Ortega Hrescak MC, Sanagua C, et al. Transrectal doppler ultrasound during prostate biopsy: Clinical utility and limitations. Actas Urol Esp 2015;39:13-19.

13. Ding Z, Ye X, Zhang L, et al. Evaluation of the performance of the ultrasound (US) elastographic Q-analysis score combined with the prostate imaging reporting and data system for malignancy risk stratification in prostate nodules based on transrectal US-magnetic resonance imaging fusion imaging. J Ultrasound Med 2019;38:29912998.

14. Kaufmann S, Kruck S, Kramer U, et al. Direct comparison of targeted MRI-guided biopsy with systematic transrectal ultrasound-guided biopsy in patients with previous negative prostate biopsies. Urol Int 2015;94:319-325.

15. Schoots IG, Roobol MJ, Nieboer D, Bangma CH, Steyerberg EW, Hunink MG. Magnetic resonance imagingtargeted biopsy may enhance the diagnostic accuracy of significant prostate cancer detection compared to standard transrectal ultrasound-guided biopsy: A systematic review and meta-analysis. Eur Urol 2015;68:438-450.

16. Wegelin O, van Melick HHE, Hooft L, et al. Comparing three different techniques for magnetic resonance imagingtargeted prostate biopsies: A systematic review of in-bore versus magnetic resonance imaging-transrectal ultrasound fusion versus cognitive registration. Is there a preferred technique? Eur Urol 2017;71:517-531.

17. Delongchamps NB, Peyromaure M, Schull A, et al. Prebiopsy magnetic resonance imaging and prostate cancer detection: Comparison of random and targeted biopsies. J Urol 2013;189:493-499.

18. Turkbey B, Rosenkrantz AB, Haider MA, et al. Prostate imaging reporting and data system version 2.1: 2019 update of prostate imaging reporting and data system version 2 . Eur Urol 2019;76:340-351.

19. Rouviere O, Puech P, Renard-Penna R, et al. Use of prostate systematic and targeted biopsy on the basis of multiparametric MRI in biopsy-naive patients (MRI-FIRST): A prospective, multicentre, paired diagnostic study. Lancet Oncol 2019;20:100-109.

20. Kasivisvanathan V, Rannikko AS, Borghi M, et al. MRItargeted or standard biopsy for prostate-cancer diagnosis. N Engl J Med 2018;378:1767-1777.

21. Oderda M, Faletti R, Battisti G, et al. Prostate cancer detection rate with Koelis fusion biopsies versus cognitive biopsies: A comparative study. Urol Int 2016;97: 230-237.

22. Yamada Y, Shiraishi T, Ueno A, et al. Magnetic resonance imaging-guided targeted prostate biopsy: Comparison between computer-software-based fusion versus cognitive fusion technique in biopsy-naive patients. Int J Urol 2020; 27:67-71.

23. Monda SM, Vetter JM, Andriole GL, et al. Cognitive versus software fusion for MRI-targeted biopsy: Experience before and after implementation of fusion. Urology 2018;119:115-120.

24. Baco E, Rud E, Eri LM, et al. A randomized controlled trial to assess and compare the outcomes of two-core prostate biopsy guided by fused magnetic resonance and transrectal ultrasound images and traditional 12-core systematic biopsy. Eur Urol 2016;69:149-156.

25. Puech P, Rouviere O, Renard-Penna R, et al. Prostate cancer diagnosis: Multiparametric MR-targeted biopsy with cognitive and transrectal US-MR fusion guidance versus systematic biopsy-Prospective multicenter study. Radiology 2013;268:461-469.

26. Galante Romo MI, Ciappara Paniagua M, Moreno Sierra J. [Fusion biopsy. Analysis of fusion software platforms.]. Arch Esp Urol 2019;72:794-803.

27. Onofrey JA, Staib LH, Sarkar S, et al. Learning non-rigid deformations for robust, constrained point-based registration in image-guided MR-TRUS prostate intervention. Med Image Anal 2017;39:29-43.

28. Mai Z, Yan W, Zhou Y, et al. Transperineal templateguided prostate biopsy: 10 years of experience. BJU Int 2016;117:424-429.

$$
\begin{array}{r}
\text { Address correspondence to: } \\
\text { Lixin Hua, PhD } \\
\text { Department of Urology } \\
\text { The First Affiliated Hospital } \\
\text { of Nanjing Medical University } \\
300 \text { Guangzhou Road } \\
\text { Nanjing } 210029 \\
\text { Jiangsu } \\
\text { China }
\end{array}
$$

E-mail: lixinhua@njmu.edu.cn

Gong Cheng, PhD Department of Urology

The First Affiliated Hospital of Nanjing Medical University 300 Guangzhou Road Nanjing 210029 Jiangsu China

E-mail: gcheng@njmu.edu.cn

$\begin{aligned} & \quad \text { Abbreviations Used } \\ & \text { BMI }=\text { body mass index } \\ & \text { bpMRI }=\text { bi-parameter MRI } \\ & \text { ciPCa }=\text { clinically insignificant prostate cancer } \\ & \text { COG-TB }=\text { cognitive fusion TB } \\ & \text { csPCa }=\text { clinically significant prostate cancer } \\ & \text { DCE }=\text { dynamic contrast enhancement } \\ & \text { DRE }=\text { digital rectal examination } \\ & \text { DWI }=\text { diffusion weighted imaging } \\ & \text { FUS-TB }=\text { software fusion TB } \\ & \text { IQR }=\text { interquartile range } \\ & \text { mpMRI }=\text { multiparametric MRI } \\ & \text { MRI }=\text { magnetic resonance imaging } \\ & \text { PI-RADS }=\text { prostate imaging reporting and data system } \\ & \text { PSA }=\text { prostate-specific antigen } \\ & \text { PSAD }=\text { PSA density } \\ & \text { PV }=\text { prostate volume } \\ & \text { TB }=\text { targeted biopsy } \\ & \text { TRUS }=\text { transrectal ultrasound }\end{aligned}$

